\title{
LA MONARQUÍA EN LA HISTORIA CONSTITUCIONAL ESPAÑOLA
}

JOAQUÍN VARELA SUANZES-CARPEGNA (1954-2018) 
SUMARIO

L. SOBERANÍA Y MONARQUÍA. 2. MONARQUÍA Y DIVISIÓN DE PODERES. 3. LA CONFIANZA REGIA Y PARLAMENTARIA DE LOS MINISTROS. 4. EL FRACASO DE LA MONARQUÍA PARLAMENTARIA EN LA HISTORIA CONSTITUCIONAL ESPAÑOLA. 5. LA MONARQUÍA PARLAMENTARIA EN LA TEORÍA CONSTITUCIONAL ESPAÑOLA. 6. APÉNDICE: LA IMPORTANCIA DE LA MONARQUÍA EN LA HISTORIA DE ESPAÑA. 


\title{
LA MONARQUÍA EN LA HISTORIA CONSTITUCIONAL ESPAÑOLA
}

\author{
JOAQUÍN VARELA SUANZES-CARPEGNA (1954-2018)
}

Catedrático de Derecho Constitucional. Universidad de Oviedo

A Alberto de la Hera, en recuerdo de nuestras cordiales discrepancias

Como su título indica, este trabajo se ocupa de la Monarquía en la historia constitucional española. Pero conviene precisar que se trata de examinar la Monarquía no sólo en los textos constitucionales, sino también en la doctrina e incluso en la práctica política.

Un examen de tan largo alcance requiere naturalmente un notable esfuerzo de síntesis. Nada mejor para ello que comenzar analizando las dos grandes cuestiones que condicionaron la concepción teórica y la articulación jurídica de la Monarquía a lo largo de nuestra historia constitucional: la soberanía y la división de poderes.

Una vez llevado a cabo este análisis, el objetivo central de este ensayo será reflexionar sobre el desarrollo de la Monarquía parlamentaria en España, tanto en la práctica constitucional como en la teoría.

\section{SOBERANÍA Y MONARQUÍA}

En relación a la decisiva cuestión de la soberanía, la doctrina constitucional española del pasado siglo se dividió en dos grandes bloques: el progresista y democrático, de un lado; el moderado y conservador, de otro. El primero de ellos hizo del principio de soberanía nacional su principal baluarte ideológico; el segundo, se mantuvo fiel al principio de «soberanía compartida» entre el Rey y las Cortes, base de la doctrina conservadora de la constitución «histórica» o «interna» de España.

Esta diferente concepción del problema de la soberanía sirve también de primera y esencial piedra de toque para distinguir las constituciones históricas españolas: mientras las de 1812, 1837 — con muchas reservas_, 1856 y 1869, recogieron el principio de soberanía nacional; El Estatuto Real de 1834 —en realidad ya antes el Estatuto de Bayona, de 1809_y las Constituciones de 1845 y 1876, se mantuvieron 
fieles a la tesis conservadora de la copartición de la soberanía entre las dos grandes instituciones decantadas por la historia de España: el Rey y las Cortes.

Esta distinta manera de plantearse el problema crucial de la soberanía tiene múltiples y decisivas consecuencias respecto de la articulación del Estado Constitucional y de su inserción en él de la Corona — nomen iuris, de la Jefatura del Estado_-, de su titular, el Rey y, por tanto, de la Monarquía como forma de Estado o de gobierno. Así ocurre, por ejemplo, en lo relativo al derecho sucesorio y al estatuto personal del Rey, a la Regencia y a la Tutela. Los textos constitucionales que recogen el principio de soberanía nacional confieren a las Cortes un papel mucho más decisivo en estos asuntos que aquellos otros que se inspiran en el principio de soberanía compartida. Pero, sin minusvalorar este asunto, voy a limitarme a conectar ahora el problema de la soberanía con el papel atribuido a la Corona en la elaboración y reforma del texto constitucional. Una atribución que determina, a su vez, la relación de la Corona de la Monarquía misma - respecto del texto constitucional.

Desde este punto de vista, la Teoría constitucional progresista y democrática, desde las Cortes de Cádiz en adelante, concibió al Monarca como un órgano constituido no constituyente, incapaz, por tanto, de participar en la elaboración y en la reforma del texto constitucional. En la práctica, el Monarca no participó en los procesos constituyentes de 1812 y 1869: en el primer caso, Femando VII estaba desterrado en Francia; en el segundo, la Corona de España estaba vacante tras el exilio de Isabel II. Pero, aun estando presentes, ni la Regente María Cristina ni su hija Isabel II participaron tampoco en la elaboración de las Constituciones de 1837 y 1856. Por otro lado, tanto la Constitución de Cádiz como la nonnata de 1856 y la de 1869, impedían que el Monarca participase, mediante su sanción y promulgación, en la reforma de la Constitución. Las Cortes, por sí solas, «decretan y sancionan» el texto fundamental. La Constitución de 1837, que en realidad era una constitución ideológicamente transaccional, a medio camino del progresismo y del moderantismo, nada dice, en cambio, sobre el papel del Rey en este importante asunto. Una omisión perfectamente calculada, que permitió a los moderados «legalizar» la reforma constitucional de 1845 (pues «legitimada» ya estaba desde los esquemas de la soberanía compartida y de la Constitución histórica).

La exclusión del Monarca de la elaboración y sobre todo — pues éste es el dato jurídico más relevante- de la reforma constitucional, tiene unas consecuencias jurídicas de enorme importancia sobre la concepción de la Monarquía. Esta, en efecto, por mor de esta exclusión y, en definitiva, del principio de soberanía nacional, deja de ser una «Monarquía constitucional» en sentido estricto — en sentido amplio lo es al ser la Constitución el origen y el límite de su poder- para convertirse en una «Monarquía democrática», como se reconocería abiertamente en 1869. Para decirlo de otro modo: la Monarquía dejaba de ser forma de Estado para ser tan sólo forma de gobierno. Una forma de gobierno que, naturalmente, podía modificarse con plena licitud jurídica si las Cortes — protagonistas del proceso constitucional— así lo entendían conveniente en el futuro. 
La doctrina moderada y conservadora, desde Jovellanos a Cánovas del Castillo, pasando por MARTÍNEZ DE LA ROSA, DONOSO CORTÉS, ALCALÁ GALIANO y PACHECO, partirá en cambio de la soberanía compartida entre el Rey y las Cortes, como una pieza esencial — sin duda la más esencial— de la doctrina de la Constitución «histórica» o «interna» de España. Una doctrina que hundía sus raíces en la vieja teoría preliberal de las leyes fundamentales y que, tras plasmarse en el Estatuto de Bayona, JOVELLANOS reformularía entre 1810 y 1811, para ser más tarde el sustento legitimador del Estatuto Real y de las Constituciones de 1845 y 1876. De acuerdo con esta doctrina, la soberanía no residía en el ente abstracto Nación, sino en dos instituciones concretas, decantadas por la historia de España: el Rey y las Cortes. O bien, como expuso hábilmente Cánovas del Castillo, la soberanía nacional en España no era otra cosa que la copartición del poder público entre esas dos grandes instituciones, que eran el pilar sobre el que se asentaba la «Monarquía moderada» o «constitucional» española. Una forma de Estado - y no sólo de gobierno- que era consustancial a la «esencia», como decía JOVELLANOS, o al «alma», como remacharía CÁNOVAS, de la «Constitución histórica o interna» de España. Una Constitución material, claro está, desde la cual era necesario partir a la hora de elaborar y reformar el documento constitucional o «constitución formal», posterior e inferior a la Constitución histórica. De este modo, la elaboración y reforma de la Constitución debía hacerse de común acuerdo entre el Rey y las Cortes, como en efecto se hizo en 1834, 1845 y 1876: las Cortes aprobando y el Monarca sancionando dichos textos constitucionales, que se limitaban a reactualizar las antiguas leyes de la Monarquía, como señalaban los Proemios de las dos primeras.

Esta decisiva participación de la Corona en la elaboración y reforma del texto constitucional guarda un estrecho vínculo con la naturaleza jurídica de la Monarquía. Esta, como ya queda dicho, no se concibe como mera forma de gobierno, de suyo mudable, sino como forma de Estado permanente, de tal suerte que la supresión de la Monarquía o su reducción a mera forma de gobierno, por «legal» que fuese, sería considerada siempre «ilegítima». La Monarquía que nace de estas premisas es, ciertamente, constitucional tanto en un sentido amplio como en sentido estricto: la Monarquía es una forma de Estado pactada o paccionada entre el Rey y las Cortes. No es consecuencia de un texto constitucional, fruto del ejercicio de un poder constituyente, sino que el texto constitucional es, en rigor, consecuencia tanto del poder regio como — de ahí su diferencia con la Monarquía germánica del siglo XIx, basada en el principio monárquico- del poder de las Cortes.

\section{MONARQUÍA Y DIVISIÓN DE PODERES}

El principio de división de poderes fue, sin duda, aceptado por toda la teoría constitucional española del siglo XIX. Este principio, sin embargo - excepto en lo relativo al poder judicial, cuya posición es básicamente la misma desde la Constitu- 
ción de Cádiz a la de 1876—, se interpretó de forma muy diferente y se plasmó también de forma muy distinta en los diversos textos constitucionales de la pasada centuria. Además, en este caso no resulta tan fácil establecer una línea diferenciadora entre el constitucionalismo progresista y democrático, de un lado, y el moderado y conservador, de otro, como ocurre con el problema de la soberanía.

En realidad, en lo tocante a este principio, es preciso distinguir, primero, entre la Constitución de Cádiz y todas las demás y, en segundo lugar — sólo en segundo lugar- entre las moderadas y conservadoras, de un lado, y las progresistas y democráticas, de otro. Comenzando por la primera distinción, resulta necesario recordar que el texto de 1812 respondía a una interpretación muy rígida del principio de división de poderes, no muy distinta de la que se había plasmado antes en la Constitución de los Estados Unidos de América y en la Constitución francesa de 1791. Los recelos ante el poder ejecutivo, tan típicos del siglo XviII, el influjo de MONTESQUIEU, ROUSSEEAU y SIEYES, así como el desconocimiento o falseamiento del constitucionalismo británico, muy alejado del mecanicismo característico del constitucionalismo continental y americano, llevaron a los constituyentes gaditanos a defender y plasmar en el texto de 1812 una división muy rígida entre los dos principales poderes del Estado: el ejecutivo y el legislativo. Si el primero se atribuye al Monarca, el segundo se confiere a las Cortes. Bien es verdad que mientras a éstas se les permite interferir en el poder ejecutivo, al Rey se le otorga una débil participación en el poder legislativo (sobre todo con una sanción de las leyes que incluía un veto meramente suspensivo), de tal forma que las Cortes se convertían en el órgano central sobre el que recae la dirección política del Estado. Los constituyentes gaditanos, en realidad, concibieron al Monarca como a un enemigo de las Cortes y, por tanto, de la Nación, que, sin embargo, era preciso mantener con el objeto de no provocar la reacción de las fuerzas contrarias al nuevo orden político, social y económico plasmado en la Constitución. A resultas de todo ello, este código vertebraba una forma de gobierno sui generis, alejada en cualquier caso del sistema parlamentario de gobierno. Así, en efecto, el texto de 1812 no preveía la existencia de un órgano colegiado de Gobierno, sino tan sólo los «Secretarios de Despacho», nombrados y separados libremente por el Rey y responsables penalmente, pero no políticamente, ante las Cortes. Por otro lado, la Constitución establecía la incompatibilidad entre el cargo de Ministro y la condición de Diputado y prohibía la disolución regia de las Cortes.

Las Constituciones posteriores, del signo que fuesen, rompen con la rigidez del texto doceañista al articular el principio de división de poderes, de tal forma que a partir de ellas resultaría posible parlamentarizar la Monarquía. Desde el Estatuto Real hasta la Constitución de 1876, se acepta la disolución regia de las Cortes y la compatibilidad entre el cargo de Ministro y la condición de Diputado, permitiendo, así, la responsabilidad política, y no sólo penal, del Gobierno ante las Cortes. Una responsabilidad que regularían en algún caso los Reglamentos parlamentarios y las prácticas constitucionales e incluso, parcialmente, el texto constitucional de 1869. Natural- 
mente, de aquí no se desprendía una concepción parlamentaria de la Monarquía, como se verá más adelante, pero sí la posibilidad de su parlamentarización.

Ciertamente, la doctrina y los textos progresistas y democráticos, de un lado, y los moderados o conservadores, de otro, difieren en lo que atañe a la participación de la Corona en la función legislativa. Si el constitucionalismo moderado y conservador concede al Monarca el veto absoluto de las leyes, el progresista y democrático (nuevamente con la excepción del texto de 1837), le concede solamente un veto meramente suspensivo. Asimismo, mientras las Constituciones moderadas y conservadoras conciben al Senado como un órgano parcialmente designado por el Rey, las progresistas y democráticas lo articulan como un órgano electivo (1856 y 1869) o mixto (1837).

El constitucionalismo progresista y democrático seguirá también unas pautas diversas a las del constitucionalismo moderado y conservador en lo que concierne a la participación de la Corona en la función ejecutiva. Las Constituciones de 1834, 1845 y 1876 (a las que debe sumarse en este punto la de 1837), atribuyen al Rey la titularidad del Poder ejecutivo, que ejercerá con «SUS» Ministros. En cambio, la de 1856 y sobre todo la de 1869 pretenden distinguir entre la Jefatura del Estado y el poder ejecutivo, siguiendo a CONSTANT, a THIERS y a PREVOST-PARA DOLL, así como al constitucionalismo inglés y belga. El ejemplo más acabado es el artículo 35 de la Constitución de 1869 que atribuye el poder ejecutivo al Rey, pero señalando que lo ejercerá «a través de sus Ministros».

En resumen, pues, si tras la muerte de Femando VII se produjo un acuerdo de gran importancia a la hora de separarse de los rígidos esquemas doceañistas, no era menos patente la disparidad de criterios a la hora de interpretar el principio de división de poderes y, en particular, el papel del Monarca y de las Cortes en el seno del Estado constitucional. En el caso del constitucionalismo moderado, esta interpretación partía de la concepción del Monarca como «nervio del Estado», presente de JOVELLANOS a CÁNOVAS, mientras que en la doctrina progresista y democrática, siempre propensa a ampliar el papel de las Cortes, la interpretación del principio de división de poderes se hizo concibiendo al Monarca como un «poder moderador»y arbitral que debía reinar, pero no gobernar.

\section{LA CONFIANZA REGIA Y PARLAMENTARIA DE LOS MINISTROS}

¿Qué ocurre con el nombramiento y cese de los Ministros? Esta decisiva cuestión recibe un idéntico tratamiento desde el código de 1812 hasta el de 1876. Corresponde al Monarca nombrar y separar «libremente» a los Ministros. A todos los Ministros. Si aquél es inviolable, estos son jurídica y políticamente responsables de los actos del Rey, que serán nulos si no llevan el refrendo ministerial. Los Ministros, pues, según 
las disposiciones constitucionales vigentes a lo largo de nuestro siglo XIX, dependen de la confianza del Rey. Exclusivamente.

Ahora bien, pese a este mismo tratamiento jurídico, las interpretaciones fueron diversas. Durante la segunda etapa de vigencia de la Constitución de Cádiz, esto es, en el Trienio Constitucional, los sectores más radicales del liberalismo, haciendo caso omiso del texto constitucional, exigieron que Fernando VII se plegara de facto a los requerimientos de las Cortes a la hora de formar Gobierno. No obstante, la opinión mayoritaria, representada por MARTINEZ DE LA ROSA y AGUSTÍN DE ARGÜELLES, se mostró partidaria de respetar el tenor literal de la Constitución.

Durante la Monarquía isabelina, tanto los moderados como los progresistas siguieron aceptando que la formación y el cese del Gobierno era una prerrogativa que la Corona debía ejercer libremente, pero ahora insistían en que ésta debía tener en cuenta la composición de las Cámaras, sobre todo la del Congreso de los Diputados. Se trataba de la doctrina de la «doble confianza», ciertamente mucho más coherente con el postulado moderado de la soberanía compartida entre el Rey y las Cortes y con la idea del Monarca como «nervio del Estado» que con el principio progresista de la soberanía nacional y con una concepción del Rey como simple «poder moderador»o arbitral de las instituciones.

La doctrina de la «doble confianza» la seguiría sustentando toda la teoría constitucional conservadora en la segunda mitad del siglo xix y en el primer tercio del actual, desde CÁNOVAS hasta SÁNCHEZ DE TOCA, aunque en la práctica, como se dirá más adelante, la confianza regia pesó siempre más que la parlamentaria.

La teoría democrática, en cambio, sobre todo desde el último tercio del siglo pasado, insistió en que fuese la voluntad del Congreso de los Diputados - y, en definitiva, la del electorado- y no las preferencias personales del Monarca, el único punto de referencia que tuviese en cuenta la Corona a la hora de nombrar y cesar al Primer Ministro, mientras que los demás componentes del Gobierno debían ser nombrados por el Rey de acuerdo con el criterio de su Presidente.

\section{EL FRACASO DE LA MONARQUÍA PARLAMENTARIA EN LA HISTORIA CONSTITUCIONAL ESPAÑOLA}

El examen de la soberanía y de la división de poderes, permite, pues, conocer con bastante precisión la concepción de la Corona en la Teoría constitucional y su posición en cada una de las Constituciones aprobadas en nuestro pasado, desde la de 1812 hasta la de 1876. Ahora bien, como se deduce de lo hasta aquí expuesto, para conocer la posición de la Corona en la historia constitucional y la naturaleza de la Monarquía resultante no basta — siendo ello imprescindible - conocer su posición en los textos constitucionales —o en otros textos normativos, como los Reglamentos parlamentarios-y su concepción en la doctrina iuspublicista, sino que es preciso conocer también cual fue su papel en el proceso político. 
Ello es particularmente cierto si se quiere saber cuál fue el grado de parlamentarización de la Monarquía española, esto es, si desde la Monarquía articulada en Cádiz hasta la que se configura en la Constitución de 1876 es posible hablar de una Monarquía parlamentaria. Al fin y al cabo, como es bien sabido, es consustancial a esta forma de Monarquía que la función de gobierno o de dirección política del Estado se desplace del Monarca, en quien reside según la Constitución, a un Gobierno responsable, exclusivamente, ante el Parlamento y, en definitiva, ante el cuerpo electoral. Un desplazamiento que se llevó a cabo históricamente en toda Europa mediante convenciones y simples prácticas políticas, esto es, mediante un proceso extrajurídico o, al menos, al margen del derecho escrito. De ahí que para conocer la posición del Rey en esta forma de gobierno no basta con tener en cuenta su status jurídico, sino que es preciso averiguar también su participación efectiva en el sistema político en cada concreta etapa histórica. La Monarquía Parlamentaria, en realidad, es tanto un producto del derecho como de la historia, siendo consustancial a ella el divorcio entre la realidad jurídica y la realidad política o, al menos, entre el derecho escrito y el derecho consuetudinario. Su estudio obliga, así, y el caso de España no es una excepción, a conjugar constantemente el análisis jurídico-constitucional con el histórico-constitucional o, para decirlo más gráficamente, a rellenar los conceptos de Teoría del Estado con historia constitucional.

¿Hasta dónde llegó el proceso de parlamentarización de la Monarquía española del ochocientos? Sin duda, a partir sobre todo de la muerte de Femando VII se produjo una indudable parlamentarización de la Monarquía constitucional española, poniéndose en práctica algunos de los mecanismos que caracterizan al «gobierno de mayoría», como se llamó el sistema parlamentario de gobierno durante la primera mitad del siglo XIX, o al «régimen parlamentario», expresión más utilizada en la segunda mitad de la pasada centuria. Si bien la distinción entre la Jefatura del Estado y el «Consejo de Ministros» o Gobierno no llegó nunca a formalizarse en los textos constitucionales — ni siquiera en 1869- dentro del Ejecutivo se fue conformando un órgano colegiado, el Gobierno, en el que sobresale un Primer Ministro, responsable ante las Cortes, sobre todo ante el Congreso de los Diputados. Es indudable también que desde el Estatuto Real hasta la Dictadura de Primo de Rivera se fueron perfeccionando los mecanismos de control parlamentario de la acción gubernamental, tanto los ordinarios (Discurso del Trono o de la Corona y su contestación, debate de los Presupuestos, preguntas, interpelaciones y comisiones de investigación), como los extraordinarios, incluida la moción de censura y el voto de confianza o «cuestión de Gabinete», como en principio se llamó entre nosotros. Unos mecanismos regulados tanto en los Reglamentos de las Cámaras como en las prácticas parlamentarias. Correlativamente, y con el objeto de equilibrar las relaciones entre el Ejecutivo y las Cortes, estas últimas fueron no pocas veces disueltas por aquel antes de que expirase su mandato.

Ahora bien, a diferencia de lo que ocurre en una auténtica Monarquía parlamentaria, en nuestra historia constitucional no fue el Parlamento $-\mathrm{y}$ en definitiva el 
electorado—, quien determinó la composición del Gobierno, sino que fue éste quien, con la anuencia regia, controló al Parlamento mediante la sistemática corrupción electoral. Una corrupción que se hizo todavía mayor tras la definitiva implantación del sufragio universal masculino, a partir de 1890.

Pero sobre todo interesa destacar que durante todo el siglo pasado la función de gobierno siguió residiendo básicamente en el Monarca, no sólo de iure - con la excepción relativa del texto constitucional de 1869- sino también de facto, incluso durante la vigencia de esta Constitución, pues si bien Amadeo I fue un Rey neutral, no dejó de ser un rey activo. No fue el Gobierno, con el respaldo de la mayoría parlamentaria y bajo la dirección de un Primer Ministro, el que entre nosotros ejerció las funciones que la Constitución atribuía al Monarca, sino que éste - ya fuese personalmente, como ocurrió con Alfonso XII y sobre todo con Alfonso XIII, o mediante una camarilla, como ocurrió con María Cristina e Isabel II- tuvo un papel muy activo en la dirección del Estado, nombrando y separando libremente a los Ministros, con independencia de sus apoyos parlamentarios, designando a Senadores, disolviendo las Cortes e interviniendo directamente en la toma de algunas decisiones de gran importancia, relativas a las relaciones entre el Estado y la Iglesia, al Ejército — particularmente con Alfonso XII y sobre todo con Alfonso XIII- y a la política internacional.

El Monarca, pues, no se limitó a formalizar decisiones adoptadas y refrendadas por los miembros del Gobierno y especialmente por su Presidente — como ocurre en una auténtica Monarquía parlamentaria, en donde el Gobierno es responsable exclusivamente ante el Parlamento de tales decisiones-, sino que tuvo una intervención a veces decisiva en la dirección política del Estado. Cuando el Monarca no ejerció la función de gobierno no fue porque esta función la ejerciese un Gabinete responsable ante las Cortes, sino porque estas últimas se arrogaron el ejercicio de tal función, como ocurrió durante el Trienio, con lo cual la Monarquía se deslizó, no por una senda parlamentaria, sino asamblearia o convencional.

En definitiva, desde las Cortes de Cádiz hasta la Dictadura de Primo de Rivera no se articuló nunca en España una auténtica Monarquía parlamentaria o, dicho de otra forma, la parlamentarización de la Monarquía constitucional no fue lo suficientemente intensa como para que pueda hablarse de una Monarquía parlamentaria española a lo largo del siglo xIX. Incluso en los breves períodos en que estuvieron en el poder las fuerzas más proclives a la parlamentarización de la Monarquía, no es posible hablar, en rigor, de una Monarquía parlamentaria. Esta forma de Monarquía no llegó nunca a establecerse entre nosotros, en efecto, ni durante el trienio esparterista, ni durante el bienio progresista, ni siquiera durante el sexenio democrático, pese a que el texto constitucional que rigió jurídicamente este último período, esto es, el de 1869, supuso el más importante éxito en la lucha por parlamentarizar (e incluso por democratizar, cosas distintas aunque relacionadas), la Monarquía constitucional a lo largo del siglo XIX, no sólo en España, sino incluso en toda Europa. 
No está de más advertir, sin embargo, que la intervención de la Corona en la función de gobierno no se debió sólo, ni principalmente, a una especie de intrusismo crónico del Monarca en la vida política española ni a una falta de respeto por parte de aquél a los usos parlamentarios —evidente en Fernando VII, pero no así, o al menos no del mismo modo, en sus sucesores - sino sobre todo a la debilidad de la sociedad española y, más exactamente, a su incapacidad para sostener un sistema estable de partidos, articulados en tomo a unos dirigentes con capacidad de liderazgo. La Restauración representa en este sentido un indudable avance: a diferencia de lo que había ocurrido durante la Monarquía isabelina, en la que el partido moderado había monopolizado el poder, CÁNOVAS consiguió crear un sistema bipartidista. Pero este sistema, que se descompuso a comienzos del reinado de Alfonso XIII, no era expresión de la sociedad, sino creación artificial del poder. Ante la ausencia de un sistema de partidos estable y maduro y de una opinión pública formada, los teóricos de la Monarquía isabelina y sobre todo de la Restauración trataron de justificar, no sin cinismo, la intervención de la Corona en la interpretación de la voluntad nacional, sustituyendo así a las Cortes.

Es necesario señalar que el fracaso de la Monarquía Parlamentaria en la práctica constitucional española, no fue óbice para que existiese una teoría constitucional, elaborada por un sector muy cualificado del liberalismo español, que reivindicó esta forma de gobierno. Como precursor de esta teorización es preciso citar a Blanco-White, quien no dejó de criticar en «El Español» el modo en que los constituyentes gaditanos interpretaron el principio de soberanía nacional y el de división de poderes. Blanco-White, en contrapartida, defendió en este periódico una Monarquía constitucional muy parlamentarizada, de acuerdo con el ejemplo suministrado por el constitucionalismo inglés y por algunos autores a los que presta particular atención, como Paley y Burke.

\section{LA MONARQUÍA PARLAMENTARIA EN LA TEORÍA CONSTITUCIONAL ESPAÑOLA}

En el Trienio Constitucional el Conde de Toreno, separándose tanto de los «exaltados», partidarios de una interpretación de la Constitución doceañista en clave asamblearia, como de los «moderados», proclives a una interpretación presidencialista del texto gaditano, defendió algunos mecanismos básicos del sistema parlamentario de gobierno, como la necesidad de un enlace entre el Gobierno y la mayoría de las Cortes. Pero esta defensa, que no tuvo entonces demasiado eco, no permite incluirle entre los teóricos de la Monarquía parlamentaria, al incardinarse en los esquemas de la doctrina de la doble confianza, contraria a esta forma de gobierno, según queda dicho. Una doctrina que defenderían tanto los moderados como la mayor parte de los progresistas durante la primera mitad del siglo XIX. 
El progresismo de izquierda, que nace tras la transacción doctrinal de 1837, tampoco quiso acogerse a los principios básicos de la Monarquía parlamentaria. Joaquín María LÓPEZ, por ejemplo, sin duda el más destacado teórico de esta corriente durante los años cuarenta y cincuenta del pasado siglo, sigue aferrado a una concepción doceañista —y, por tanto, no parlamentaria- de la Monarquía, en virtud de la cual pretende anular las prerrogativas constitucionales de la Corona, no mediante su traspaso fáctico a un Gobierno salido de las Cortes, sino a través de subordinar el ejecutivo —esto es, el Monarca y «SUS» Ministros— a la voluntad de la representación nacional.

En realidad, será preciso esperar a los debates de las Constituyentes de 1869 para hallar el germen de lo que puede calificarse, con cierto rigor, de teoría de la Monarquía parlamentaria. Una teoría que, ahora ya de forma más sistemática y clara, sostuvieron durante la Restauración algunos demócratas todavía partidarios de la Monarquía, como Gumersindo DE AZCÁRATE, GINER DE LOS RÍOS, SANTAMARÍA DE PAREDES y Adolfo POSADA. Intelectuales todos ellos vinculados, de una y otra forma, al krausismo, y los dos últimos profesores en activo de Derecho Político. De todos ellos fue AZCÁRATE, sin embargo, el que sostuvo de forma más recurrente y constante una teoría democrática de la Monarquía parlamentaria, atacando al núcleo de la teoría moderada y conservadora de la Monarquía constitucional: la doctrina de la soberanía compartida, la idea del Monarca como nervio del Estado y, por consiguiente, la teoría de la doble confianza. Frente a tales tesis, AZCÁRATE sostuvo la doctrina progresista de la soberanía nacional y la concepción del Rey como poder moderador y arbitral, defendiendo tenazmente que sólo la confianza del Parlamento - de un Parlamento elegido limpiamente por sufragio universal debería ser el órgano ante el que Gobierno rindiese cuentas de su gestión.

\section{APÉNDICE: LA IMPORTANCIA DE LA MONARQUÍA EN LA HISTORIA DE ESPAÑA}

No quisiera terminar este estudio sin unas reflexiones finales acerca del decisivo papel que desempeñó la Corona en el constitucionalismo español del siglo XIX. Unas reflexiones que deben ser tenidas en cuenta no a modo de conclusión de todo cuanto hasta aquí se ha dicho, sino más bien a modo de apéndice.

El liberalismo doceañista, muy radical si se compara con el que le sucedió, no fue, sin embargo, republicano. Faltó entre nosotros entonces un movimiento jacobino y republicano. En plena acefalia de la Monarquía, y pese al desprestigio de los borbones después del motín de Aranjuez y de las renuncias de Bayona, se pide unánimemente la vuelta de Fernando VII, el «deseado».

Pese a la represión feroz que este llevó a cabo contra el liberalismo, tanto en 1814 como sobre todo en 1823, ni durante el Trienio ni a partir de 1834 el republicanismo representa algo serio en España. La Monarquía sigue, en cambio, siendo aceptada por casi todos: desde luego por los carlistas, que seguirán defendiéndola, al modo tradi- 
cional, en los campos de batalla en tres ocasiones durante el siglo xix, pero también por los liberales.

Los moderados harán de ella, como se ha dicho ya, el centro de la teoría y de la práctica del Estado Constitucional, desde MARTÍNEZ DE LA ROSA hasta CÁNOVAS DEL CASTILLO, desde el Estatuto Real hasta la Constitución de 1876, desde la Regente María Cristina hasta Alfonso XIII. Los progresistas e incluso los demócratas - la mayoría de ellos monárquicos o al menos no republicanos- seguirían defendiendo la Monarquía, incluso la borbónica, pese a la actitud sectaria de la Regente María Cristina y de su hija Isabel II.

Cierto que progresistas y demócratas exigen tras la revolución de 1868 la abdicación de Isabel II, pero no por ello cambian sus convicciones, que siguen siendo monárquicas. La I República no nace de derribar la Monarquía, como había ocurrido en la Inglaterra de Cronwell y en la Francia de 1793 y 1848, sino por la abdicación de un Rey, Amadeo I de Saboya, harto de no poder ser un rey pasivo — pues neutral lo fue siempre-. Y tras la Restauración, CÁNOVAS logra que casi todos los progresistas y demócratas que habían apoyado la revolución de 1868 e incluso que no pocos y muy cualificados republicanos, como CASTELAR, acepten e incluso apoyen, con un entusiasmo digno de conversos, la Monarquía borbónica restaurada.

Sólo en 1931 el pueblo español fue capaz de derribar la Monarquía y de proclamar la República. Pero, como es sabido, por poco tiempo. Cabe concluir, pues, que el único régimen que ha superado el trauma histórico de la ausencia de un Monarca hereditario en la Jefatura del Estado ha sido el de la dictadura militar del General Franco. Un éxito, ciertamente, que sólo se explica a condición de que tengamos en cuenta que el dictador se convirtió en un Monarca y su régimen en una Monarquía. Una Monarquía absoluta, desde luego, y no constitucional, en el sentido liberal de este término.

Una Monarquía absoluta que no fue sustituida tampoco por una República, sino por una Monarquía, ciertamente democrática, pero en la que el Rey, y no el pueblo, actúo como «motor del cambio» durante la transición democrática y como «defensor de la Constitución» cuando la democracia peligró el 23 de febrero de 1981. La Monarquía se impuso en el proceso de transición política, como se había impuesto antes —aunque de una forma distinta - en el proceso de 1876. Sirvió de puente o de enlace entre el franquismo y la democracia. Era un legado que había que aceptar si se quería que la transición no fuese abortada por la fuerza de las armas —esto es, por las Fuerzas Armadas - y ello explica también la protección que recibe en la Constitución de 1978, según la cual la reforma del estatuto personal del Rey, por mínimo que sea, se somete a un procedimiento mucho más complejo que la reforma del Tribunal Constitucional, un órgano jurídicamente mucho más importante, pero que no lo es desde el punto de vista político. 